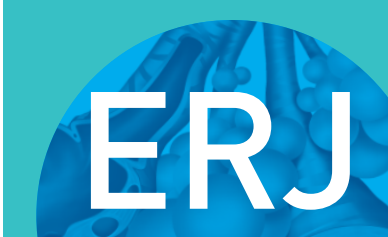

open research
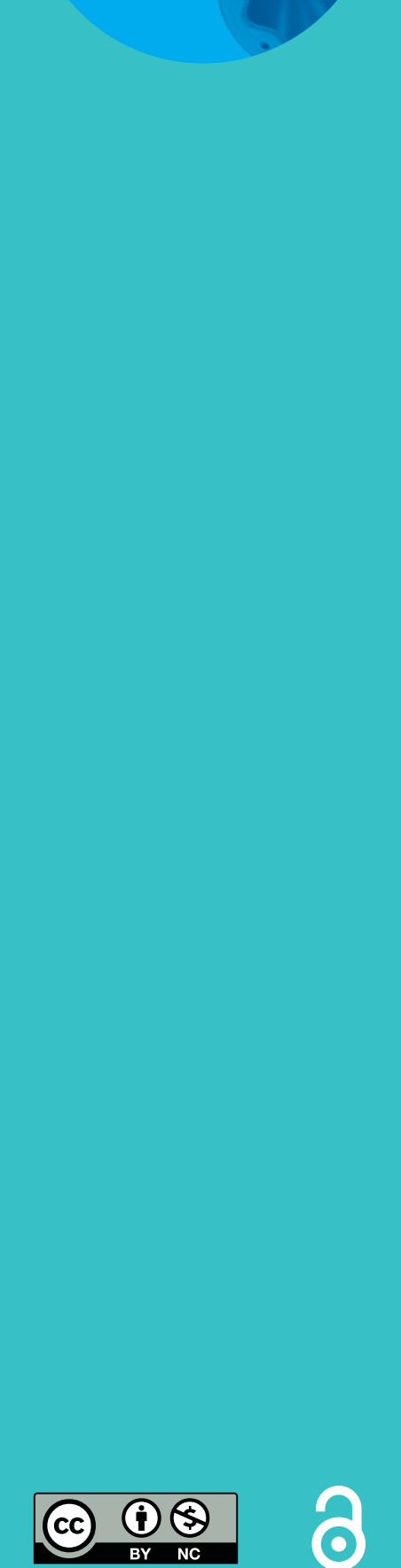

\section{Relationship between dietary patterns and COPD: a systematic review and meta-analysis}

\author{
Michael K. Parvizian (10 ${ }^{1}$, Manreet Dhaliwal ${ }^{1}$, Jeremy Li $^{1}$, Imran Satia ${ }^{2,3}$ and \\ Om P. Kurmi (1) ${ }^{2,4}$
}

Affiliations: ${ }^{1}$ Dept of Medicine, McMaster University, Hamilton, ON, Canada. ${ }^{2}$ Dept of Medicine, Division of Respirology, McMaster University, Hamilton, ON, Canada. ${ }^{3}$ Faculty of Biology, Medicine and Health, Division of Infection, Immunity and Respiratory Medicine, University of Manchester, Manchester, UK. ${ }^{4}$ Population Health Research Institute, McMaster University, Hamilton, ON, Canada.

Correspondence: Om P. Kurmi, Population Health Research Institute, Hamilton Health Sciences, 237 Barton St East, Hamilton, Ontario, Canada. E-mail: kurmiodmcmaster.ca

\section{ABSTRACT}

Background: Findings from previous studies reporting on the associations between chronic obstructive pulmonary disease (COPD) and various dietary patterns have been inconsistent. This review aims to summarise the evidence on the strength of the association between dietary patterns and the prevalence and incidence of COPD.

Methods: We conducted a comprehensive search of seven databases between 1 January 1980 and 30 November 2019. Two reviewers independently reviewed each manuscript through the screening, selection, data extraction and quality assessment stages. Data from eight observational studies that met the inclusion criteria were extracted and random-effects meta-analysis was subsequently conducted.

Results: Eight observational studies (all eight reporting on healthy dietary patterns and three on unhealthy dietary patterns) met the inclusion criteria and data were extracted to include in the meta-analysis. Consumption of a healthy dietary pattern was associated with a lower risk of COPD (pooled OR 0.88, 95\% CI 0.82-0.94). Consumption of unhealthy dietary patterns was associated with a higher risk of COPD (OR 1.22, 95\% CI 0.84-1.76); however, the results were not statistically significant and had high heterogeneity $\left(\mathrm{I}^{2}=91 \%\right)$.

Conclusion: Our results suggests that healthy dietary patterns are associated with a lower prevalence of COPD, while unhealthy dietary patterns are not. More studies, particularly adequately powered longitudinal studies, are needed to further elucidate the effects of healthy and unhealthy dietary patterns on risk of COPD.

@ERSpublications

This review suggests that individuals with healthy dietary pattern have lower risk of chronic obstructive pulmonary disease http://bit.ly/331PVJ1

Cite this article as: Parvizian MK, Dhaliwal M, Li J, et al. Relationship between dietary patterns and COPD: a systematic review and meta-analysis. ERJ Open Res 2020; 6: 00168-2019 [https://doi.org/ 10.1183/23120541.00168-2019].

This article has supplementary material available from openres.ersjournals.com

The protocol for this review is registered at https://www.crd.york.ac.uk/prospero/ with identifier number CRD42018094503.

Received: 2 July 2019 | Accepted after revision: 28 Feb 2020

Copyright $\odot$ ERS 2020. This article is open access and distributed under the terms of the Creative Commons Attribution Non-Commercial Licence 4.0. 


\section{Introduction}

Chronic obstructive pulmonary disease (COPD) is the third leading cause of death worldwide and is associated with poor quality of life (QoL) and a significant burden on healthcare resources [1, 2]. Current population-level strategies to reduce the burden of COPD have largely focused on smoking cessation, the most significant modifiable risk factor for the development and progression of COPD [3]. However, recent studies have reported a high burden of COPD among nonsmokers, which has supported the search for other modifiable risk factors in COPD development and progression. Studies have identified other factors, such as diet, socioeconomic status, air pollution and occupational exposures, that may play a role in the development and progression of COPD [4-6].

Several systematic reviews have reported that specific dietary components, such as high fatty acid content or consumption of high dietary fibre, may be protective against COPD [5]. Additionally, large population-based studies have reported that the consumption of a high fruit and vegetable diet rich in antioxidants is associated with decreased COPD [6-8]. As people do not consume specific foods or nutrients in isolation, there has been a shift towards studying the health effects of dietary patterns (e.g. the Mediterranean Diet and the Western Diet), rather than individual nutrients in isolation. Furthermore, dietary patterns are more easily interpreted and understood by the public allowing, for easier translation into COPD management $[9,10]$.

A prior systematic review did investigate the association between dietary patterns and COPD [11] However, this review was limited by a small number of studies, which required pooling prevalence with incidence and loosely defining dietary patterns to include several studies of specific foods [11]. A meta-analysis was conducted but interpretation was limited due to significant heterogeneity $\left(\mathrm{I}^{2}>70 \%\right)$ amongst the studies. However, since then, several new studies have been published, possibly providing enough data for a meta-analysis [11]. Additionally, research has recently demonstrated the importance of dietary patterns on other relevant outcomes such as spirometric indices, and a review in this area has not yet been conducted [12].

Therefore, we conducted a systematic review to investigate the effects of various dietary patterns on the prevalence of COPD. Additionally, we sought to capture secondary outcomes if available, including the incidence of $\mathrm{COPD}$, spirometric indices (forced expiratory volume in $1 \mathrm{~s}\left(\mathrm{FEV}_{1}\right)$, forced vital capacity $(\mathrm{FVC})$ and $\left.\mathrm{FEV}_{1} / \mathrm{FVC}\right)$, COPD mortality and QoL in those already diagnosed with COPD.

\section{Methods}

We conducted and reported this systematic review according to the Preferred Reporting Items for Systematic Review and Meta-analysis guidelines [13]. The protocol for this review was registered with PROSPERO (identifier number CRD42018094503).

\section{Data sources and search strategy}

Database searches were conducted in MEDLINE, Embase, Web of Science, CINAHL, LILACS, AMED and the Cochrane database for articles published between 1 January 1980 and 30 November 302019 using the key terms described in tables S1-S5 for each database. Search terms were selected to encompass articles reporting on dietary patterns and their relationship to COPD. Hand searches of the reference lists of published systematic reviews and recently published articles were also conducted to identify additional articles for inclusion in this review.

\section{Study selection}

We included studies investigating the effect of dietary patterns on COPD outcomes in both adults and children. We included studies that met our predefined inclusion criteria: 1) randomised controlled trial, cross-sectional, case-control or cohort studies; 2) studies reporting associations between COPD prevalence, incidence, QoL assessed with a validated tool, spirometric lung function or mortality and at least one dietary pattern; and 3) studies that defined COPD by either physician diagnosis or objective spirometric testing $\left(\mathrm{FEV}_{1} / \mathrm{FVC}<0.7\right.$ or lower limit of normal criteria).

We excluded studies that defined diet based on individual nutrients, foods or food groups, as well as studies in which the presence of self-reported symptoms alone defined COPD. We also excluded studies that were not available in English.

Following initial calibration exercises, teams of two reviewers (two of M.K. Parvizian, M. Dhaliwal and J. Li) independently screened titles and abstracts for inclusion in the analysis. Subsequently, any articles judged as potentially meeting the inclusion criteria underwent a full-text review. All discrepancies were resolved by consensus and a third reviewer was consulted if disagreement was not resolved between the two reviewers. If authors published multiple papers on the same dietary pattern and outcomes in the same 
cohort, the study with the longest follow-up was selected. Inter-rater reliability was assessed for each data screening pair using Cohen's $\kappa$ at both screening stages.

\section{Data extraction and quality assessment}

A data extraction form was developed for this review using the Covidence platform [14]. The predefined data extraction form included author, year of publication, study design, number of total participants, number of cases, age, sex, dietary assessment method, factors adjusted for in the analysis, effect estimates and confidence intervals. Whenever a study reported more than the one adjusted estimate of effect for an outcome, we chose the most adjusted effect estimate.

Two reviewers (M.K. Parvizian and M. Dhaliwal) independently grouped each dietary pattern into one of three categories based on the makeup of each diet (i.e. foods and nutrients consumed): healthy, unhealthy or neutral. The Healthy Eating Index 2010 criteria were used to assess patterns [15]. The foods and nutrients included in relevant dietary patterns were assessed, and the overall pattern categorised by the reviewers. Healthy diets would have high levels of fruit, vegetables, beans and green vegetables, whole grains, dairy, seafood and plant proteins, and fatty acids, coupled with low levels of refined grains, sodium and empty calories, with unhealthy diets not meeting these criteria [15]. Several dietary patterns were identified: cosmopolitan, traditional, refined-foods, high-carbohydrate, Dutch dietary guidelines, Mediterranean, prudent, Western, Alternate Healthy Eating Index (AHEI), American Heart Association (AHA) and Dietary Approaches to Stop Hypertension (DASH). Of these diets, the Mediterranean, prudent, DASH, AHEI, Dutch dietary guidelines, cosmopolitan and AHA were classified as healthy, whereas Western, refined-foods, high-carbohydrate and traditional were classified as unhealthy [16-22]. None of the investigated dietary patterns fell into the neutral category. All disagreements were resolved by consensus. Dietary patterns are summarised in table S6 for each study.

Teams of two reviewers (two of M.K. Parvizian, M. Dhaliwal and J. Li) independently assessed the risk of bias and methodological quality of each study included in the review, using the National Institutes of Health Quality Assessment Tools (NIH-QATs) [23, 24].

\section{Data synthesis and analysis}

Statistical analyses and figure generation was performed using Cochrane's Review Manager 5.3 and RStudio (utilising the meta, metafor and dmetar packages) $[25,26]$. For all statistical tests performed, a p-value $<0.05$ was considered significant.

The studies included in this review reported outcomes comparing the prevalence or incidence of COPD in different tertiles, quartiles or quintiles of dietary pattern adherence scores. The analysis was performed using dichotomous outcomes comparing the highest adherence group and lowest adherence group. Dichotomous outcomes were analysed using pooled odds ratios or risk ratios (RRs) with 95\% confidence intervals, where sufficient data were available for meta-analysis. Studies reporting hazard ratios were only included in the meta-analysis if enough data to calculate the odds ratio or RR were included. Studies investigating multiple dietary patterns in one study population had the subgroups within the study combined prior to meta-analysis, so that the population was not inaccurately weighted in the forest plot [27]. As this meta-analysis included only nonrandomised studies, random-effects models were calculated using a generic inverse variance method to allow for the most appropriately adjusted odds ratio to be included [28].

To evaluate heterogeneity, we calculated Q-statistics and the inconsistency index $\left(\mathrm{I}^{2}\right)$ for each pooled statistic. We considered an $\mathrm{I}^{2}$ of $25 \%, 50 \%$ and $75 \%$ to represent low, moderate and high degrees of heterogeneity, respectively [29]. To investigate heterogeneity, subgroup analyses were performed based on geographic region, age, specific dietary pattern and sex where data permitted. Metaregression was performed in analyses with $\geqslant 10$ studies [30].

To assess publication bias, a Begg's funnel plot was generated for any outcome with $\geqslant 10$ included studies [31]. An Egger's test was performed to quantify asymmetry [32]. The trim-and-fill method was utilised if funnel plot asymmetry was suspected [32-35].

\section{Results}

\section{Study selection}

Our search and selection criteria are summarised in figure 1. Of the 12730 studies identified through various database searches, 2894 were duplicates and 9661 did not meet the inclusion criteria for full-text review. Out of the remaining 175 studies that were fully reviewed, data were extracted from the 12 articles that met all the inclusion criteria. No studies reported on QoL or mortality. 


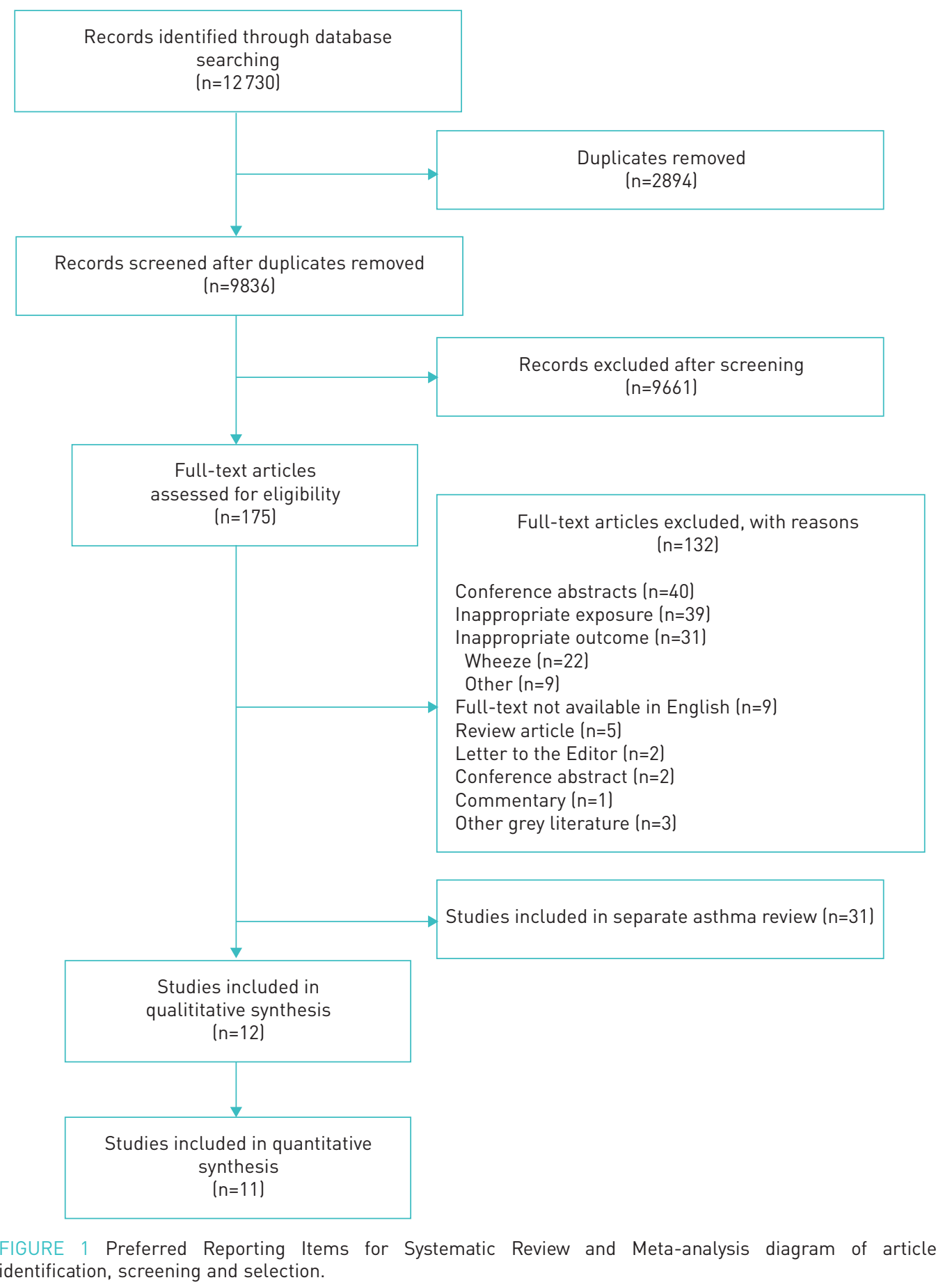

There was a high degree of agreement at each level of the review between the three pairs of reviewers with Cohen's $\kappa$ ranging from 0.85 to 0.88 at the title and abstract screening level, and ranging from 0.86 to 0.91 at the full-text screening level.

\section{Characteristics of included studies}

Evidence for this review came from 12 studies: nine cohort studies, two case-control studies and one cross-sectional study. However, all but five of these studies performed a cross-sectional analysis (i.e. calculated an OR). The characteristics of the included studies are presented in table 1. All included studies investigated COPD in adults (age range 20-75 years) and were from Europe $(n=6)$, the USA $(n=5)$ or the Middle East $(n=1)$. All studies collected their dietary data using food frequency questionnaires, which 


\section{TABLE 1 Characteristics of included studies}

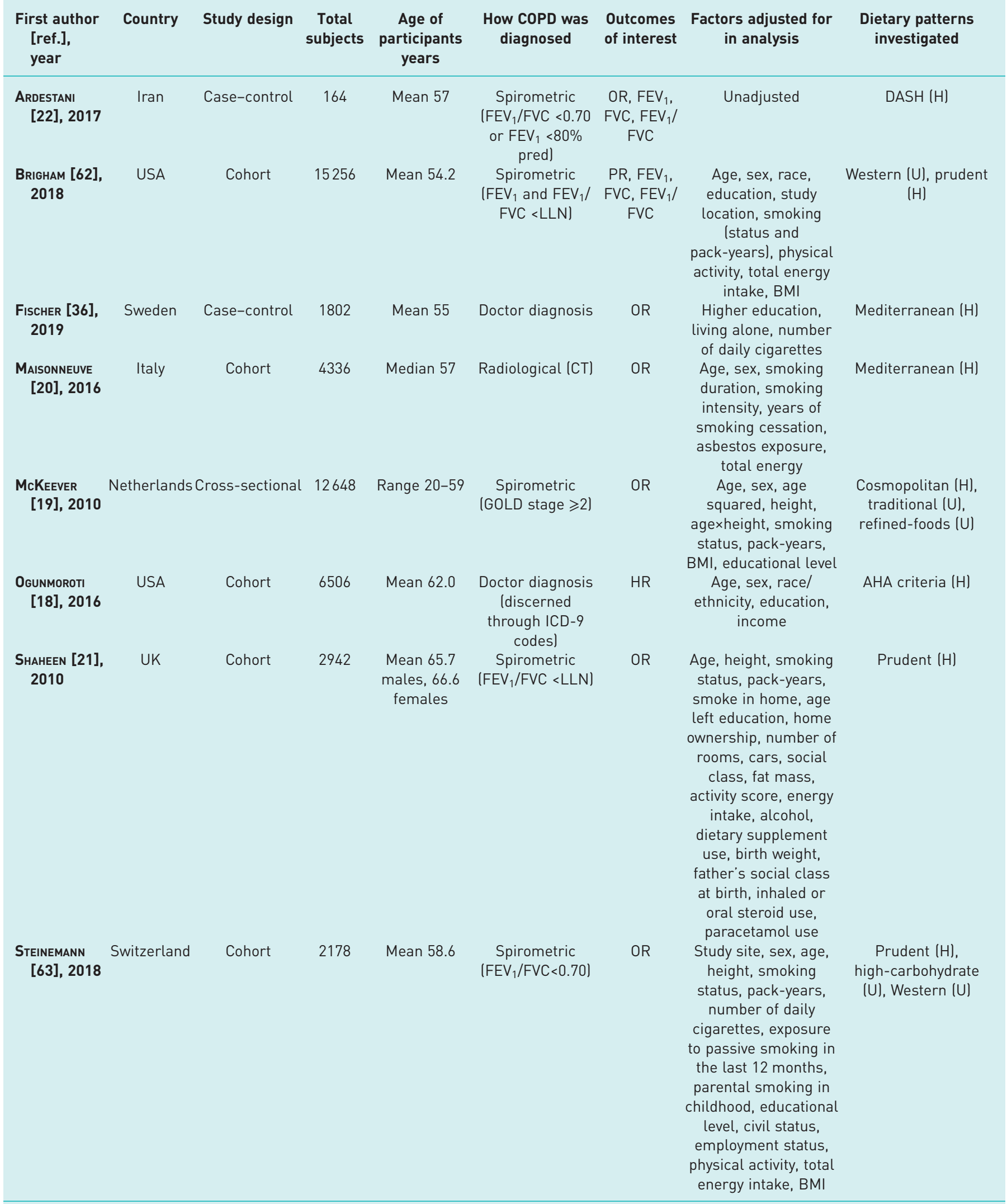




\section{TABLE 1 Continued}

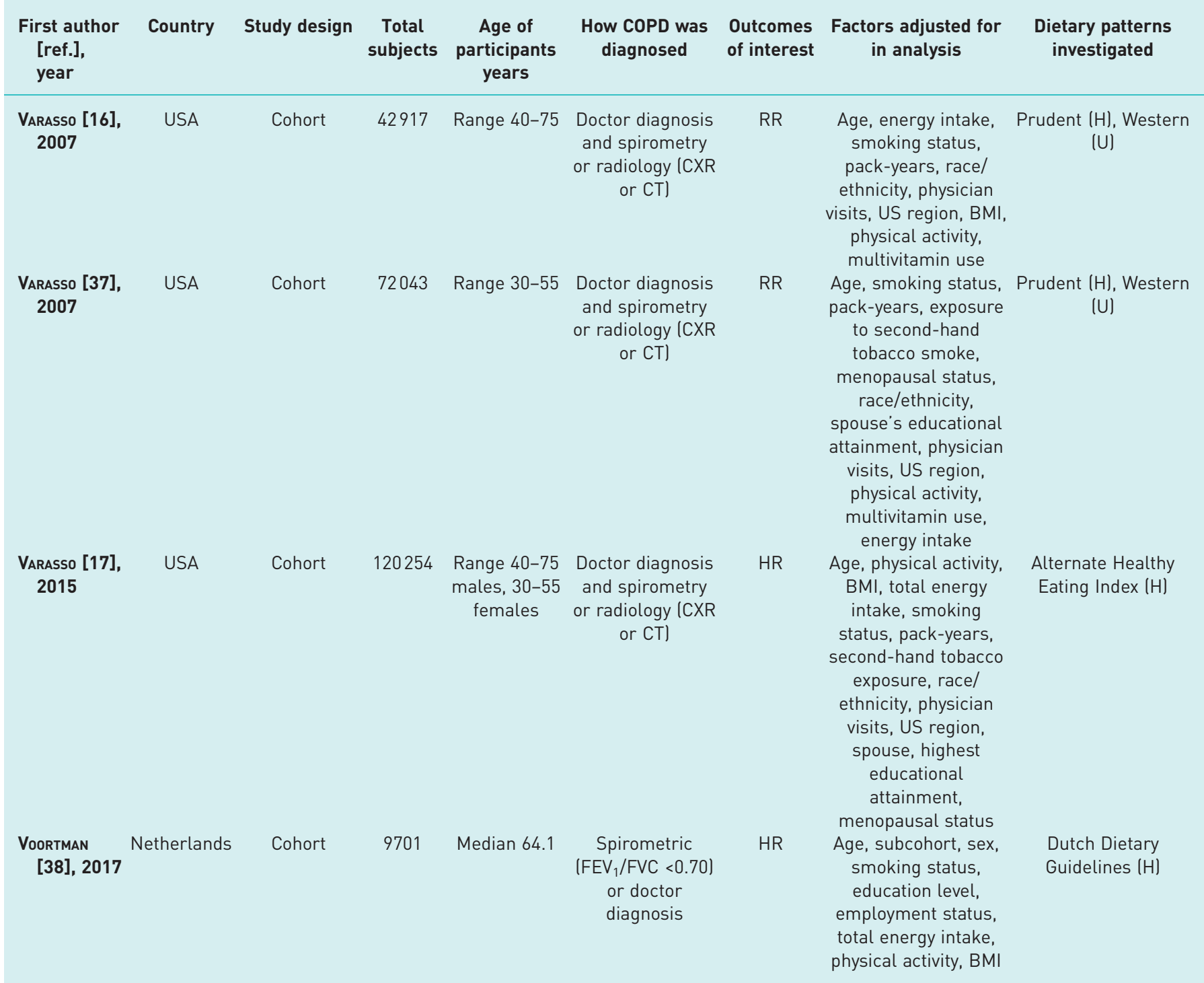

FEV $_{1}$ : forced expiratory volume in $1 \mathrm{~s}$; FVC: forced vital capacity; LLN: lower limit of normal; CT: computed tomography; GOLD: Global Initiative for Chronic Obstructive Lung Disease; ICD-9: International Classification of Diseases, ninth revision; CXR: chest radiography; PR: prevalence ratio; HR: hazard ratio; RR: risk ratio; BMI: body mass index; DASH: Dietary Approaches to Stop Hypertension; H: healthy; U: unhealthy; AHA: American Heart Association; AHEI: Alternative Healthy Eating Index.

varied between studies. Most studies derived dietary patterns using principal component analysis. A full description of each dietary pattern and the method used to define the patterns included in our review is provided in table S6.

Quality assessments of studies (cohort and cross-sectional) are presented in table S7. Based on the overall quality rating of these 10 studies, four were determined to be "good", five "fair" and one "poor". The other two included studies were case-control studies by ARDESTANi et al. [22] and Fischer et al. [36], which were evaluated using a different NIH-QAT checklist and were thus not included in the table. The study by ARDESTANi et al. [22] was deemed to be of overall "fair" quality, with several concerns: it was unclear if all eligible cases and/or controls were invited to participate, it was unclear whether dietary exposure occurred prior to the development of COPD and there was uncertain blinding status of assessors. The study by FISCHER et al. [36] was deemed to be of overall "fair" quality, with several concerns, including that there was no sample size justification, there was no clear use of concurrent controls and it was unclear whether dietary exposure occurred prior to COPD development. 


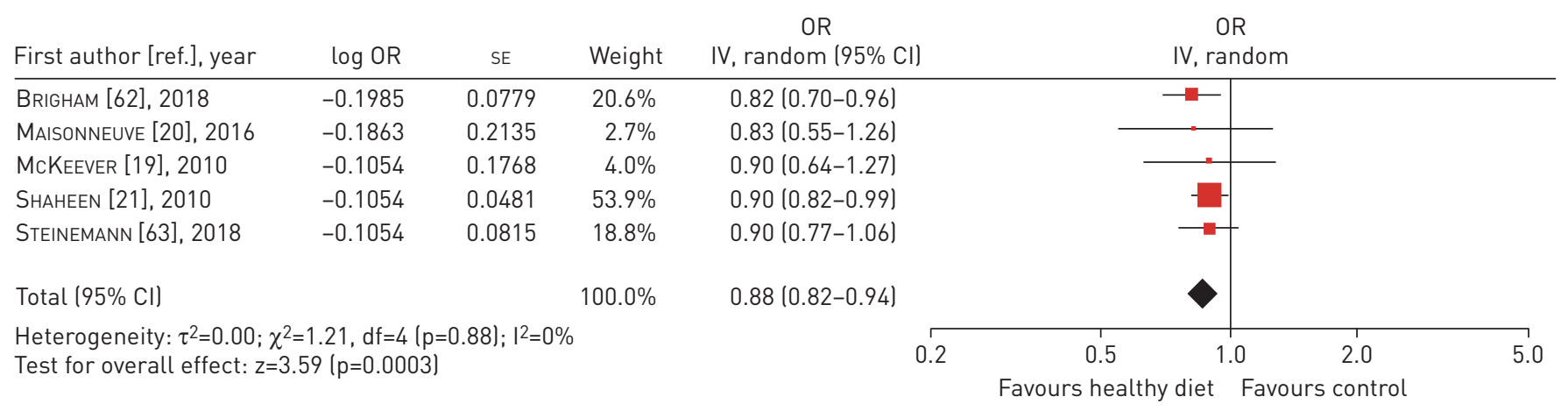

FIGURE 2 Forest plot of healthy dietary patterns and prevalence of COPD in cross-sectional studies. IV: inverse variance; df: degrees of freedom. Error bars represent $95 \%$ confidence intervals.

\section{Healthy dietary patterns and COPD}

The pooled effect estimates of five cross-sectional analyses (figure 2) (OR 0.88, 95\% CI 0.82-0.94) and three cohort studies (figure 3) (RR 0.56, 95\% CI 0.37-0.84) suggested a lower risk of COPD in those adhering to a healthy dietary pattern. However, while the cross-sectional studies had minimal heterogeneity $\left(\mathrm{I}^{2}=0 \%\right)$, there was significant heterogeneity $\left(\mathrm{I}^{2}=78 \%\right)$ among the cohort studies. Of the studies in this meta-analysis, it is important to note that VARraso et al.[16] and VARraso et al. [37] investigated only males and females respectively. Subgroup analysis or investigation of potential publication bias was not conducted due to insufficient study numbers.

While eligible for inclusion in our review, Ogunmoroti et al. [18] and Voortman et al. [38] were not included in this meta-analysis as there were insufficient data provided to calculate odds ratios or RRs based on the HR. Ogunmoroti et al. [18] reported that the ideal diet according to the AHA was not significantly associated with new-onset of COPD compared to a poor diet (HR 0.44, 95\% CI 0.06-3.21), while Voortman et al. [38] demonstrated that following the Dutch dietary guidelines was associated with reduced COPD (HR 0.94, 95\% CI 0.91-0.98).

\section{Unhealthy dietary patterns and COPD}

Five studies, four cohort and one cross-sectional, investigated the association between unhealthy dietary patterns and the development of COPD. Meta-analysis (figure 4) of three studies reported a nonsignificant increase in the prevalence of COPD (OR 1.14, 95\% CI 0.87-1.50) for those adhering to an unhealthy diet. However, there was significant heterogeneity $\left(\mathrm{I}^{2}=85 \%\right)$. Subgroup analysis or investigation of potential publication bias was not conducted due to insufficient study numbers. A meta-analysis of longitudinal studies investigating the association between unhealthy dietary patterns and COPD was not performed as there were only two studies. These studies demonstrated a statistically significant increase in COPD incidence (OR 4.56, 95\% CI 1.95-10.70) [16] and a nonsignificant increase in COPD incidence (OR 1.31, 95\% CI 0.94-1.82) [37].

\section{Dietary patterns and spirometric outcomes}

ARDESTANi et al. [22] reported the mean per cent of predicted spirometric values for cases and controls based on tertiles of adherence to a DASH diet. There was no significant association between DASH tertiles

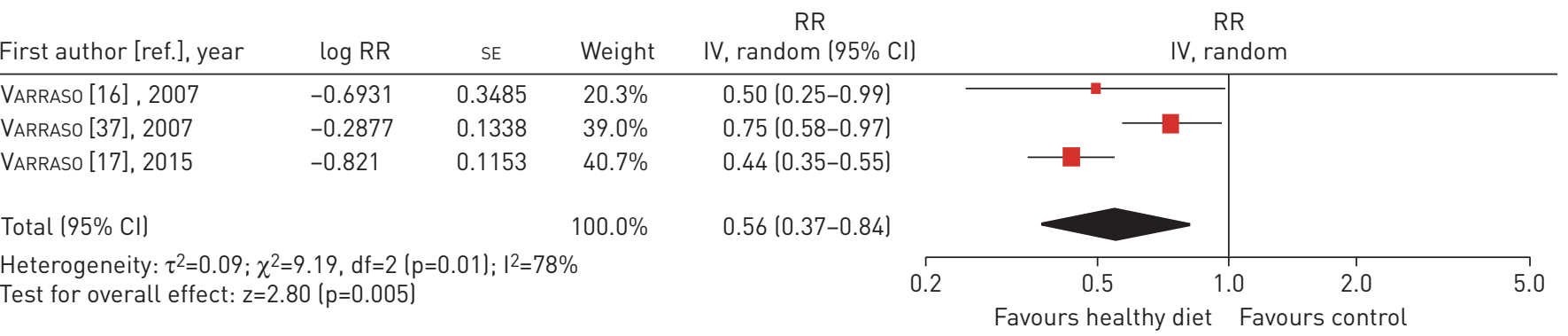

FIGURE 3 Forest plot of healthy dietary patterns and incidence of COPD in cohort studies. RR: risk ratio; IV: inverse variance; df: degrees of freedom. Error bars represent $95 \%$ confidence intervals. 


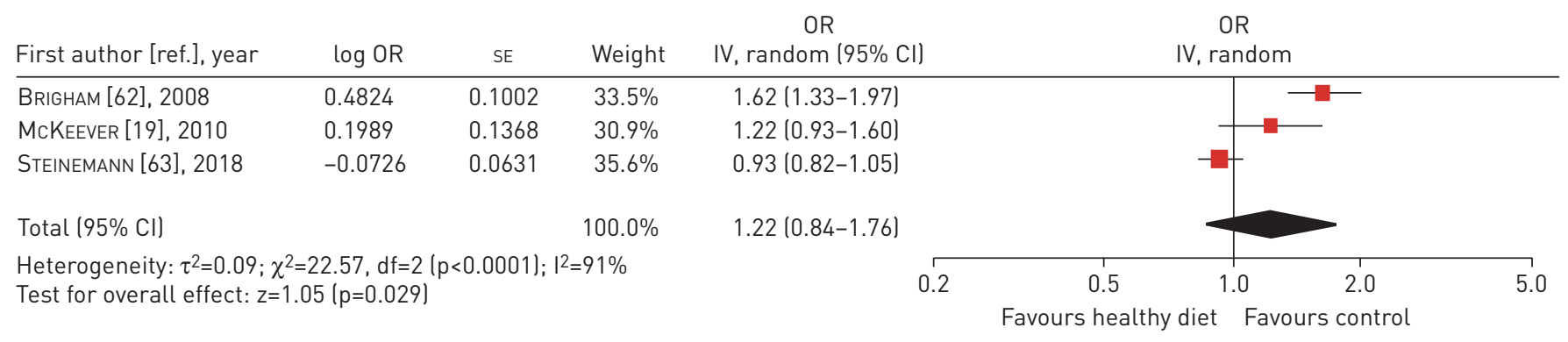

FIGURE 4 Forest plot of unhealthy dietary patterns and prevalence of COPD in cross-sectional studies. IV: inverse variance; df: degrees of freedom. Error bars represent $95 \%$ confidence intervals.

and spirometric outcomes in those with COPD, although a significant decrease in the $\mathrm{FEV}_{1} / \mathrm{FVC}$ ratio was noted in those with higher adherence to the DASH diet amongst controls $(\mathrm{p}=0.04)$.

\section{Discussion}

While previous research has mainly investigated the effects of individual nutrients and food groups on COPD, the investigation of the combined effects of overall dietary patterns is an important area of ongoing research. This systematic review summarises the findings from observational studies published to date on the association between dietary patterns and COPD. Overall, adherence to a healthy dietary pattern was associated with a lower prevalence of COPD, whereas adhering to an unhealthy dietary pattern was associated with a higher prevalence of COPD (statistically not significant).

The aim of this study was to investigate the risk of different dietary patterns at an epidemiological level. The exact mechanism by which diet affects risk of COPD is not known; however, several possible explanations have been proposed: 1) alteration of systemic oxidant and antioxidant balance [9, 39-41]; 2) changing the function of various inflammatory proteins and receptors $[42,43]$ (e.g. polyunsaturated fatty acids can impact the functioning of interferon- $\gamma$ receptors) [41, 44]; 3) nutrients being metabolised into pro- or anti-inflammatory molecules and thus modulating disease risk [45]; 4) changes in the gut microbiota [46]; or 5) food containing saturated fatty acids, fibre and vitamin $\mathrm{C}$ affecting lung volumes, respiratory muscle strength and gas exchange [21, 47-52].

Even though the mechanism is not well understood, meta-analysis showed a greater association between adherence to a healthy diet and decreased COPD prevalence or incidence. However, there were only three studies that looked at the relationship longitudinally as most cohort studies had an embedded cross-sectional comparison and had high heterogeneity, limiting the interpretability of the latter summary statistic. The main reasons for this was likely due to very different inclusion criteria; one study recruited females subjects between 30 and 55 years of age while another study recruited only males between the ages of 40 and 75 years. Furthermore, the third study used the same two cohorts from the previous two studies but looked at a different dietary pattern, thus not resolving the intrinsic differences between the first two study groups. Thus, the meta-analysis of healthy dietary pattern adherence and COPD incidence is essentially based only on two cohorts with important key differences in baseline characteristics.

While there were insufficient studies to perform subgroup analysis, the studies by Varraso and colleagues $[16,37]$ investigated the effects of western diet in men and women, and reported an increase in COPD risk in both men and women, although the result was not statistically significant in women. These results, taken together, may point towards an overall lower risk of COPD development in females, but previous research contradicts this finding [53]. One potential explanation for this trend is a difference in other key risk factor exposures that may not have been fully controlled for between men and women in the various studies, such as exposure to pollution, socioeconomic status, and other associated lifestyle interventions like exercise.

Our findings suggest consumption of unhealthy dietary patterns was associated with higher risk of COPD; however, the findings were not statistically significant and had high heterogeneity. While subgroup analysis and investigation for publication bias would be ideal to explore potential causes of this heterogeneity, this was not possible due to insufficient study numbers. There are several potential causes that may explain the heterogeneity evident in this analysis. Firstly, the various dietary patterns included in this analysis included the Western Diet $(n=2)$, traditional diet $(n=1)$, refined-foods diet $(n=1)$ and high-carbohydrate diet $(n=1)$. The exact foods contained within each dietary pattern, even amongst the two labelled as "Western Diet", varied between studies and this is a potential source of significant heterogeneity in this analysis. Additionally, different geographic regions were evident between studies with two studies conducted in 
western Europe and one in the USA. Publication bias is another key potential source of heterogeneity, which we were unable to explore due to insufficient study numbers.

Although several studies looked at the relationship between dietary patterns and spirometric indices, these studies looked at mixed populations that had participants with and without COPD. Only ARDESTANI et al. [22] separated populations by the presence or absence of COPD. They found that there was a significant relationship between healthy dietary patterns and spirometric indices in controls but not cases. However, this was a small case-control study, so it is difficult to draw any conclusions.

Strengths of our review include the methodological rigour used to conduct this study compared to prior reviews on the topic. Although there has already been a review published on this topic by ZHENG et al. [11], a strength of our review is that we were more stringent on the definition of COPD, requiring a physician, radiological and/or pulmonary function testing-based diagnosis, reducing the risk of misclassification and potential bias as a result. We additionally excluded studies that investigated oligonutrients or individual foods, which we did not consider to be dietary patterns. This led to the exclusion of eight out of 13 studies included in the prior review due to their analysis of COPD in relation to vegetable/fruit intake $(n=3)$, cured meat intake $(n=2)$, fibre intake $(n=2)$ or cured meat and vegetables/ fruit separately $(n=1)[11,54-61]$. Only five of the studies were deemed to investigate true dietary patterns [11]. Another strength of our study is the rigorous search methodology used, in particular, our attempt to identify all potential studies for inclusion by our use of numerous databases and broad search terms. Finally, this review had enough data to avoid pooling of different outcomes, which led to less heterogeneity than previous reviews.

There were several key limitations to our study. First, most studies included were conducted in Europe or North America, potentially reducing the applicability of study findings to other populations. It is also possible that studies not published in English were excluded. Hence, the results may not be consistent in other regions where indoor or outdoor air pollution may strongly influence the development of COPD, or the relative scarcity of food during childhood may affect lung development. Secondly, our review had a considerable degree of heterogeneity $\left(\mathrm{I}^{2}>0.75\right)$ for the meta-analyses investigating healthy diets and the incidence of COPD, as well as unhealthy diets and the prevalence of COPD. The cause of this is likely to be multifactorial including different study populations, different methods of dietary information collection between studies, potential publication bias, biological sex differences between studies, and the different dietary patterns included under the broad umbrella categories of "healthy" versus "unhealthy". The pooling of different dietary patterns based upon healthy and unhealthy was a methodological limitation of this review but there were not enough studies to look at individual dietary patterns separately. Thirdly, due to the observational nature of our review, it is difficult to infer causality.

Our review holds important implications for future clinical practice. The previous review by ZHENG et al. [11] did conclude that healthy dietary patterns reduced the risk of COPD, while unhealthy dietary patterns led to increased risk. While useful for hypothesis generation, the inclusion of studies not investigating true dietary patterns made the translation of results into clinical practice challenging. Additionally, the significant heterogeneity in their meta-analyses limited the interpretability of their findings. Repeating this systematic review and meta-analysis with strict inclusion criteria, we were able to replicate their findings in a cross-sectional analysis of healthy diets with minimal heterogeneity. Future research using well-powered but longitudinal designs and randomised controlled trials investigating the effects of well-characterised dietary patterns on the incidence of COPD is needed to bridge the existing gaps in knowledge. Additional cohort studies will allow investigation of potential causes or sources of heterogeneity as well as the potential for publication bias; this is paramount and necessary for the accurate interpretation of the overall body of evidence. Additional research looking at other important outcomes in COPD (i.e. exacerbations, spirometry, QoL and mortality) and the cumulative effects of confounding exposures associated with COPD (i.e. pollution, sex, physical activity, occupational exposures and socioeconomic status) is also needed and will be useful in translating current research into future clinical practice.

Conflict of interest: None declared.

\section{References}

1 Ahmed M, Neyaz A, Aslami A. Health-related quality of life of chronic obstructive pulmonary disease patients: Results from a community based cross-sectional study in Aligarh, Uttar Pradesh, India. Lung India 2016; 33: $148-153$.

2 Lozano R, Mohsen N, Kyle F, et al. Global and regional mortality from 235 causes of death for 20 age groups in 1990 and 2010: a systematic analysis for the Global Burden of Disease Study 2010. Lancet 2012; 380: 2095-2128.

3 Laniado-Laborin R. Smoking and chronic obstructive pulmonary disease (COPD). Parallel epidemics of the 21st century. Int J Environ Res Public Health 2009; 6 : 209-224. 
Kanervisto M, Vasankari T, Laitinen T, et al. Low socioeconomic status is associated with chronic obstructive airway diseases. Respir Med 2011; 105: 1140-1146.

5 Hanson C, Rutten EP, Wouters EFM, et al. Influence of diet and obesity on COPD development and outcomes. Int J Chron Obstruct Pulmon Dis 2014; 9: 723-733.

6 Lamprecht B, McBurnie MA, Vollmer WM, et al. COPD in never smokers: RESULTS from the population-based burden of obstructive lung disease study. Chest 2011; 139: 752-763.

7 Kaluza J, Larsson SC, Orsini N, et al. Fruit and vegetable consumption and risk of COPD: a prospective cohort study of men. Thorax 2017; 72: 500-509.

8 Hu FB. Dietary pattern analysis: a new direction in nutritional epidemiology. Curr Opin Lipidol 2002; 13: 3-9.

9 National Research Council. Diet and health: implications for reducing chronic disease risk. Washington, National Academies Press, 1989.

10 Hong JY, Lee CY, Lee MG, et al. Effects of dietary antioxidant vitamins on lung functions according to gender and smoking status in Korea: a population-based cross-sectional study. BMJ Open 2018; 8: e020656.

11 Zheng PF, Shu L, Si CJ, et al. Dietary patterns and chronic obstructive pulmonary disease: a meta-analysis. COPD 2016; 13: 515-522.

12 Gutiérrez-Carrasquilla L, Sánchez E, Hernández M, et al. Effects of Mediterranean Diet and physical activity on pulmonary function: a cross-sectional analysis in the ILERVAS Project. Nutrients 2019; 11: 329.

13 Moher D, Liberati A, Tetzlaff J, et al. Preferred Reporting Items for Systematic Reviews and Meta-Analyses: The PRISMA Statement (PRISMA 2009 Checklist). PLoS Med 2009; 6: e1000097.

14 Veritas Health Innovation. Covidence systematic review software. 2018.

15 Adjoian TK, Firestone MJ, Eisenhower D, et al. Validation of self-rated overall diet quality by Healthy Eating Index-2010 score among New York City adults, 2013. Prev Med Reports 2016; 3: 127-131.

16 Varraso R, Fung TT, Barr RG, et al. Prospective study of dietary patterns and chronic obstructive pulmonary disease among US women. Am J Clin Nutr 2007; 86: 488-495.

17 Varraso R, Chiuve SE, Fung TT, et al. Alternate Healthy Eating Index 2010 and risk of chronic obstructive pulmonary disease among US women and men: Prospective study. BMJ 2015; 350: h286.

18 Ogunmoroti O, Allen NB, Cushman M, et al. Association between life's simple 7 and noncardiovascular disease: The multi-ethnic study of atherosclerosis. J Am Heart Assoc 2016; 5: e003954.

19 McKeever TM, Lewis SA, Cassano PA, et al. Patterns of dietary intake and relation to respiratory disease, forced expiratory volume in $1 \mathrm{~s}$, and decline in 5-y forced expiratory volume. Am J Clin Nutr 2010; 92: 408-415.

20 Maisonneuve P, Shivappa N, Hébert JR, et al. Dietary inflammatory index and risk of lung cancer and other respiratory conditions among heavy smokers in the COSMOS screening study. Eur J Nutr 2016; 55: 1069-1079.

21 Shaheen SO, Jameson KA, Syddall HE, et al. The relationship of dietary patterns with adult lung function and COPD. Eur Respir J 2010; 36: 277-284.

22 Ardestani ME, Onvani S, Esmailzadeh A, et al. Adherence to Dietary Approaches to Stop Hypertension (DASH) dietary pattern in relation to chronic obstructive pulmonary disease (COPD): a case-control study. J Am Coll Nutr 2017; 36: 549-555.

23 National Heart Lung and Blood Institute. Study Quality Assessment Tools. https://www.nhlbi.nih.gov/healthtopics/study-quality-assessment-tools.

24 Higgins JPT, Altman DG, Gøtzsche PC, et al. The Cochrane Collaboration's tool for assessing risk of bias in randomised trials. BMJ 2011; 343: d5928.

25 Schwarzer G, Carpenter JR, Rücker G. Meta-analysis with R. Basel, Springer, 2015.

26 Harrer M, Cuijpers P, Furukawa TA, et al. Doing meta-analysis in R: a hands-on guide. Denver, PROTECT Lab, 2019.

27 Borenstein M, Hedges LV, Higgins JP, et al. Introduction to Meta-Analysis. Hoboken, John Wiley \& Sons, 2009.

28 Reeves BC, Deeks JJ, Higgins JPT, et al. Cochrane Handbook for Systematic Reviews of Interventions. Chichester, John Wiley \& Sons, 2008

29 Higgins JP, Thompson SG, Deeks JJ, et al. Measuring inconsistency in meta-analyses. BMJ 2003; 327: 557-560.

30 Thompson SG, Higgins JP. How should meta-regression analyses be undertaken and interpreted? Stat Med 2002 21: 1559-1573.

31 Begg CB, Mazumdar M. Operating characteristics of a rank correlation test for publication bias. Biometrics 1994; 50: 1088-1101.

32 Egger M, Smith GD, Schenider M, et al. Bias in meta-analysis detected by a simple graphical test. BMJ 1997; 315: 629-634.

33 Duval S, Tweedie R. Trim and fill: a simple funnel-plot-based method of testing and adjusting for publication bias in meta-analysis. Biometrics 2000; 56: 455-463.

34 Terrin N, Schmid CH, Lau J, et al. Adjusting for publication bias in the presence of heterogeneity. Stat Med 2003; 22: $2113-2126$.

35 Peters JL, Sutton AJ, Jones DR, et al. Performance of the trim and fill method in the presence of publication bias and between-study heterogeneity. Stat Med 2007; 26: 4544-4562.

36 Fischer A, Johansson I, Bomberg A, et al. Adherence to a Mediterranean-like Diet as a protective factor against COPD: a nested case-control study. COPD 2019; 16: 272-277.

37 Varraso R, Fung TT, Hu FB, et al. Prospective study of dietary patterns and chronic obstructive pulmonary disease among US men. Thorax 2007; 62: 786-791.

38 Voortman T, Kiefte-de Jong J, Ikram M, et al. Adherence to the 2015 Dutch dietary guidelines and risk of non-communicable diseases and mortality in the Rotterdam Study. Eur J Epidemiol 2017; 32: 993-1005.

39 Macnee W. Oxidants/antioxidants and COPD. Chest 2000; 117: 303S-317S.

40 Brutsche MH, Downs SH, Schindler C, et al. Bronchial hyperresponsiveness and the development of asthma and COPD in asymptomatic individuals: SAPALDIA Cohort Study. Thorax 2006; 61: 671-677.

41 Matsuyama W, Mitsuyama H, Watanabe M, et al. Effects of omega-3 polyunsaturated fatty acids on inflammatory markers in COPD. Chest 2005; 128: 3817-3827.

42 Feng C, Keisler DH, Fritsche KL. Dietary omega-3 polyunsaturated fatty acids reduce IFN-gamma receptor expression in mice. J Interferon Cytokine Res 1999; 19: 41-48.

43 Purasiri P, Murray A, Richardson S, et al. Modulation of cytokine production in vivo by dietary essential fatty acids in patients with colorectal cancer. Clin Sci 1994; 87: 711-717. 
Darmani H, Parton J, Harwood JL, et al. Interferon-gamma and polyunsaturated fatty acids increase the binding of lipopolysaccharide to macrophages. Int J Exp Pathol 1994; 75: 363-368.

45 Wall R, Ross RP, Fitzgerald GF, et al. Fatty acids from fish: the anti-inflammatory potential of long-chain omega-3 fatty acids. Nutr Rev 2010; 68: 280-289.

46 Maslowski KM, Mackay CR. Diet, gut microbiota and immune responses. Nat Immunol 2011; 12: 5-9.

47 Cornell K, Alam M, Lyden E, et al. Saturated fat intake is associated with lung function in individuals with airflow obstruction: results from NHANES 2007-2012. Nutrients 2019; 11: 317.

48 Schwartz J, Weiss S. The relationship of dietary fish intake to level of pulmonary function in the first National Health and Nutrition Survey (NHANES I). Eur Respir J 1994; 7: 1821-1824.

49 Van de Bool C, Rutten E, van Helvoort A, et al. A randomized clinical trial investigating the efficacy of targeted nutrition as adjunct to exercise training in COPD. J Cachexia Sarcopenia Muscle 2017; 8: 748-758.

50 Efthimiou J, Mounsey P, Benson D, et al. Effect of carbohydrate rich versus fat rich loads on gas exchange and walking performance in patients with chronic obstructive lung disease. Thorax 1992; 47: 451-456.

51 Schwartz J, Weiss ST. Relationship between dietary vitamin C intake and pulmonary function in the First National Health and Nutrition Examination Survey (NHANES). Am J Clin Nutr 1994; 9: 251-251.

52 Hanson C, Lyden E, Rennard S, et al. The relationship between dietary fiber intake and lung function in the National Health and Nutrition Examination Surveys. Ann Am Thorac Soc 2016; 13: 643-650.

53 Barnes PJ. Sex differences in chronic obstructive pulmonary disease mechanisms. Am J Respir Crit Care Med 2016 193: 813-814.

54 Watson L, Margetts B, Howarth P, et al. The association between diet and chronic obstructive pulmonary disease in subjects selected from general practice. Eur Respir J 2002; 20: 313-318.

55 Varraso R, Willett WC, Camargo CA. Prospective study of dietary fiber and risk of chronic obstructive pulmonary disease among US women and men. Am J Epidemiol 2010; 171: 776-784.

56 Varraso R, Jiang R, Barr RG, et al. Prospective study of cured meats consumption and risk of chronic obstructive pulmonary disease in men. Am J Epidemiol 2007; 166: 1438-1445.

57 Yin $\mathrm{P}$, Jiang $\mathrm{Y}$, Zhang $\mathrm{M}$, et al. Association between frequency of fruit and vegetable intake and chronic obstructive pulmonary disease. Chinese J Prev Med 2011; 45: 707-710.

58 Hirayama F, Lee AH, Binns CW, et al. Do vegetables and fruits reduce the risk of chronic obstructive pulmonary disease? A case-control study in Japan. Prev Med 2009; 49: 184-189.

59 Jiang R, Paik DC, Hankinson JL, et al. Cured meat consumption, lung function, and chronic obstructive pulmonary disease among United States adults. Am J Respir Crit Care Med 2007; 175: 798-804.

60 Kan H, Stevens J, Heiss G, et al. Dietary fiber, lung function, and chronic obstructive pulmonary disease in the atherosclerosis risk in communities study. Am J Epidemiol 2008; 167: 570-578.

61 Yang L, Lu X, Deng J, et al. Risk factors shared by COPD and lung cancer and mediation effect of COPD: two center case-control studies. Cancer Causes Control 2015; 26: 11-24.

62 Brigham EP, Steffen LM, London SJ. Diet pattern and respiratory morbidity in the Atherosclerosis Risk in Communities Study. Ann Am Thorac Soc 2018; 15: 675-682.

63 Steinemann N, Grize L, Pons M. Associations between dietary patterns and post-bronchodilation lung function in the SAPALDIA Cohort. Respiration 2018; 95: 454-463. 